\title{
Oscillatory and Asymptotic Behavior of a First-Order Neutral Equation of Discrete Type with Variable Several Delay under $\Delta$ Sign
}

\author{
Radhanath Rath $\mathbb{D}^{1,2}$ and Chittaranjan Behera $\mathbb{D}^{3}$ \\ ${ }^{1}$ VSSUT, Burla, Sambalpur, 768018 Odisha, India \\ ${ }^{2}$ Former Principal Khallikote Autonomous College, Berhampur, 760001 Odisha, India \\ ${ }^{3}$ Department of Mathematics, Silicon Institute of Technology, Bhubaneswar, Odisha, India \\ Correspondence should be addressed to Radhanath Rath; radhanathmath@yahoo.co.in
}

Received 18 July 2018; Accepted 23 September 2018; Published 10 October 2018

Academic Editor: Hans Engler

Copyright (c) 2018 Radhanath Rath and Chittaranjan Behera. This is an open access article distributed under the Creative Commons Attribution License, which permits unrestricted use, distribution, and reproduction in any medium, provided the original work is properly cited.

We obtain necessary and sufficient conditions so that every solution of neutral delay difference equation $\Delta\left(y_{n}-\sum_{j=1}^{k} p_{n}^{j} y_{n-m_{j}}\right)+$ $q_{n} G\left(y_{\sigma(n)}\right)=f_{n}$ oscillates or tends to zero as $n \longrightarrow \infty$, where $\left\{q_{n}\right\}$ and $\left\{f_{n}\right\}$ are real sequences and $G \in C(\mathbb{R}, \mathbb{R}), x G(x)>0$, and $m_{1}, m_{2}, \ldots, m_{k}$ are positive integers. Here $\Delta$ is the forward difference operator given by $\Delta x_{n}=x_{n+1}-x_{n}$, and $\left\{\sigma_{n}\right\}$ is an increasing unbounded sequences with $\sigma_{n} \leq n$. This paper complements, improves, and generalizes some past and recent results.

\section{Introduction}

Consider the neutral delay difference equation of first order

$$
\Delta\left(y_{n}-\sum_{j=1}^{k} p_{n}^{j} y_{n-m_{j}}\right)+q_{n} G\left(y_{\sigma(n)}\right)=f_{n}
$$

where $\Delta$ is the forward difference operator given by $\Delta x_{n}=$ $x_{n+1}-x_{n}, q_{n}$ and $f_{n}$ are members of infinite real sequences, and $m_{j}$ are positive integers. Further, assume $\left\{p_{n}^{j}\right\}$ are real sequences for each $j \in 1,2, \ldots k$ and that $G \in C(\mathbb{R}, \mathbb{R})$ and $\sigma(n) \leq n$ are monotonic increasing sequences which are unbounded.

We study the oscillatory behavior of solutions of neutral difference equation (1) under the following assumptions.

(H1) $x G(x)>0$ for $x \neq 0$.

(H2) There exists a bounded sequence $\left\{F_{n}\right\}$ such that $\Delta F_{n}=$ $f_{n}$.

(H3) The sequence $\left\{F_{n}\right\}$ in (H2) satisfies $\lim _{n \rightarrow \infty} F_{n}=0$.

(H4) $q_{n}>0, \sum_{n=n_{0}}^{\infty} q_{n}=\infty$.
In addition to the above we assume some new conditions on $p_{n}^{j}$ (see (12), (22), (26), and (30) in next section). It is important to note that our results hold good for the solutions of the neutral equation

$$
\Delta\left(y_{n}-\sum_{j=1}^{k} p_{n}^{j} y_{n-m_{j}}\right)+\sum_{j=1}^{l} q_{n}^{j} G\left(y_{\sigma_{j}(n)}\right)=f_{n}
$$

under the assumption

$$
\sum_{n=n_{0}}^{\infty}\left(\sum_{j=1}^{l} q_{n}^{j}\right)=\infty, \quad q_{n}^{j}>0
$$

instead of (H4). The following neutral difference equations/delay difference equations are obtained as particular case of (2).

$$
\begin{array}{r}
\Delta\left(y_{n}-p_{n} y_{n-m}\right)+\sum_{j=1}^{l} q_{n}^{j} G\left(y_{\sigma_{j}(n)}\right)=f_{n}, \\
\Delta\left(y_{n}-p_{n} y_{n-m}\right)+q_{n} G\left(y_{\sigma(n)}\right)=f_{n},
\end{array}
$$




$$
\Delta\left(y_{n}\right)+\sum_{j=1}^{l} q_{n}^{j} G\left(y_{\sigma_{j}(n)}\right)=0
$$

and

$$
\Delta\left(y_{n}\right)+q_{n} G\left(y_{\sigma(n)}\right)=0
$$

The neutral difference equations (5) are seen as the discrete analogue of the neutral differential equations

$$
(y(t)-p(t) y(t-\tau))^{\prime}+q(t) G(y(t-\sigma))=f(t) .
$$

The oscillatory and asymptotic behavior of delay difference equations and neutral difference equations have been intensively studied in recent years due to its various application in different field of science and technology [1]. It is observed that several articles (see [2-4]) exist in literature for the study of neutral difference equations/delay difference equations with several delay, i.e., for (4) or (6), respectively. However study of neutral equations with several delay term under $\Delta$ symbol, i.e., (1) or (2), seems to be relatively scarce in literature. Use of lemmas from [1, Lemma 1.5.1 and 1.5.2] or its discrete analogue (see [5]) plays an important role in studying (4) [6], (5) [7], and (8) [8]. In this context, one may note these lemmas cannot be applied to the study of (1) or (2). Hence study of (1) and (2) needs a different approach.

The work in this paper complements and generalizes the work in $[3,9]$. This can be verified that the results in $[3,9]$ which are concerned with the study of (6) and (7) cannot be applied to the delay difference equation

$$
\Delta\left(y_{n}\right)+\left(e^{-2}-e^{-3}\right) y_{n-2}=0
$$

which has a solution $y_{n}=e^{-n}$ tending to zero. It is because the primary assumption,

$$
\liminf _{k \rightarrow \infty} \sum_{i=\sigma(k)}^{k-1} q_{i}>\frac{1}{e}
$$

is not satisfied. However, note that (10) implies (H4) and (H4) is satisfied in (9) and hence the results of this paper give an answer to the behavior of solutions of neutral equations like (9). While working on nonlinear neutral equations most of the authors $[7,8,10-12]$ assume the condition that $G$ is nondecreasing unlike this paper.

Let $n_{0}$ be a fixed nonnegative integer. Let $\max \left\{m_{1}\right.$, $\left.m_{2}, \ldots, m_{k}\right\}=m$ and $\rho=\min \left\{\sigma\left(n_{0}\right), n_{0}-m\right\}$. By a solution of (1) we mean a real sequence $\left\{y_{n}\right\}$ which is defined for all positive integers $n \geq \rho$ and satisfies (1) for $n \geq n_{0}$. Clearly if the initial condition

$$
y_{n}=a_{n} \quad \text { for } \rho \leq n \leq n_{0}
$$

is given then (1) has a unique solution satisfying the given initial condition (11). A solution $\left\{y_{n}\right\}$ of (1) is said to be oscillatory if, for every positive integer $n_{0}>0$, there exists $n \geq n_{0}$ such that $y_{n} y_{n+1} \leq 0$; otherwise $\left\{y_{n}\right\}$ is said to be nonoscillatory. In the sequel, unless otherwise specified, when we write a functional inequality, it will be assumed to hold for all $n$ sufficiently large. Here we assume the existence of solution of (1) and study its oscillatory and asymptotic behavior.

\section{Sufficient Condition}

In this section we present some results which prove that (H4) is sufficient for any solution of (2) to be oscillatory or tending to zero as $n \longrightarrow \infty$. Moreover we give some examples to illustrate and signify our results. Our first result and the subsequent ones are as follows.

Theorem 1. Suppose that (H1)-(H4) hold. Assume that there exists a positive constant $p$ such that the sequences $\left\{p_{n}^{j}\right\}$ for $j=$ $1,2, \ldots, k$ satisfy the condition

$$
\begin{aligned}
& \qquad p_{n}^{j} \geq 0, \quad \text { for every } j=1,2, \ldots, k \\
& \text { and } \sum_{j=1}^{k} \limsup _{n \rightarrow \infty} p_{n}^{j}<p<1 .
\end{aligned}
$$

Then every solution of (1) oscillates or tends to zero as $n \longrightarrow$ $\infty$.

Proof. Let $y=\left\{y_{n}\right\}$ be any solution of (1) for $n \geq n_{0}$, where $n_{0}$ is a fixed positive integer. If it oscillates then there is nothing to prove; otherwise, it leads to two distinct possibilities, either $y_{n}>0$ or $y_{n}<0$. for $n \geq n_{1}>n_{0}$. Consider the first one, i.e., $y_{n}>0$ eventually. There exits positive integer $n_{2} \geq n_{1}$ such that $y_{n}>0, y_{n-m_{j}}>0$ for each $j$, and $y_{\sigma(n)}>0$ for $n \geq n_{2}$. For $n \geq n_{2}$, let

$$
z_{n}=y_{n}-\sum_{j=1}^{k} p_{n}^{j} y_{n-m_{j}}
$$

and

$$
w_{n}=z_{n}-F_{n}
$$

From (1), (13), and (14), it follows due to (H1) that

$$
\Delta w_{n}=-q_{n} G\left(y_{\sigma(n)}\right) \leq 0
$$

Then there exists $n_{3} \geq n_{2}$ such that $w_{n}$ is monotonic and is of constant sign for $n \geq n_{3}$. For the sake of a 
contradiction assume that $y_{n}$ is not bounded. Then there exists a subsequence $\left\{y_{n_{r}}\right\}$ such that

$$
\begin{aligned}
& n_{r} \longrightarrow \infty, \\
& y_{n_{r}} \longrightarrow \infty
\end{aligned}
$$

as $r \longrightarrow \infty$,

and

$$
y\left(n_{r}\right)=\max \left\{y_{n}: n_{3} \leq n \leq n_{r}\right\}
$$

Since $\sigma(n) \longrightarrow \infty$ as $n \longrightarrow \infty$, we may choose $r$ large enough so that $\sigma\left(n_{r}\right) \geq n_{3}$. For $0<\epsilon$, because of (H3), we can find a positive integer $n_{4}$ such that $n \geq n_{4} \geq n_{3}$ implies $\left|F_{n}\right|<\epsilon$. As (12) holds, then using (13), (14), and (17) we obtain

$$
\begin{aligned}
w_{n_{r}} & =y_{n_{r}}-\sum_{j=1}^{k} p_{n}^{j} y_{n_{r}-m_{j}}-F_{n_{r}} \geq\left(1-\sum_{j=1}^{k} p_{n}^{j}\right) y_{n_{r}}-\epsilon \\
& >(1-p) y_{n_{r}}-\epsilon .
\end{aligned}
$$

Taking $r \longrightarrow \infty$, we find $\lim _{n \rightarrow \infty} w_{n}=\infty$, a contradiction as $w_{n}$ is monotonic decreasing. Hence $y_{n}$ is bounded which implies $w_{n}$ and $z_{n}$ are bounded and $\lim _{n \rightarrow \infty} w_{n}$ exists. Further it follows that $\liminf _{n \rightarrow \infty} y_{n}$ and $\limsup _{n \rightarrow \infty} y_{n}$ exist. We claim lim inf $\lim _{n \rightarrow \infty} y_{n}=0$. Otherwise, let $y_{n} \geq \alpha>0$. Next boundedness of $y_{n}$ yields $y_{n} \leq \beta$. Hence we have $0 \leq$ $\alpha<y_{n} \leq \beta$, which will be used for bounding the $G$ term in (1) from below.

From the continuity of $G$ and assumption (H1) it follows that there exists a positive lower bound $m$ for $G$ on $[\alpha, \beta]$. Hence there exists $n_{5}$ such that $G\left(y_{\sigma(n)}\right)>m>0$ for $n>n_{5}$. Then summing (15) from $n=n_{5}$ to $s-1$ we obtain

$$
w_{n_{5}}-w_{s}=\sum_{j=n_{5}}^{s-1} q_{j} G\left(y_{\sigma(j)}\right) \geq m \sum_{j=n_{5}}^{\infty} q_{j} .
$$

Since the left hand side is the member of a bounded sequence, while the right hand side approaches $+\infty$, we have a contradiction. This yields $\lim \inf _{n \rightarrow \infty} y_{n}=0$. From (H3), monotonic nature of $w_{n}$ and (14), it follows that $\lim _{n \rightarrow \infty} z_{n}$ exists finitely. Let $\lim _{n \rightarrow \infty} z_{n}=\delta$. If $\delta>0$, then

$$
\begin{aligned}
0 & <\delta=\liminf _{n \longrightarrow \infty} z_{n}=\liminf _{n \longrightarrow \infty}\left(y_{n}-\sum_{j=1}^{k} p_{n}^{j} y_{n-m_{j}}\right) \\
& \leq \liminf _{n \longrightarrow \infty} y_{n}+\limsup _{n \longrightarrow \infty}\left(-\sum_{j=1}^{k} p_{n}^{j} y_{n-m_{j}}\right)
\end{aligned}
$$

$$
\begin{aligned}
& =-\liminf _{n \longrightarrow \infty}\left(\sum_{j=1}^{k} p_{n}^{j} y_{n-m_{j}}\right) \leq-\sum_{j=1}^{k} \liminf _{n \longrightarrow \infty}\left(p_{n}^{j} y_{n-m_{j}}\right) \\
& \leq-\sum_{j=1}^{k}\left(\liminf _{n \longrightarrow \infty} p_{n}^{j}\right)\left(\liminf _{n \longrightarrow \infty} y_{n-m_{j}}\right) \leq 0,
\end{aligned}
$$

a contradiction. If $\delta \leq 0$ then

$$
\begin{aligned}
& 0 \geq \delta=\limsup _{n \rightarrow \infty} z_{n}=\limsup _{n \rightarrow \infty}\left(y_{n}-\sum_{j=1}^{k} p_{n}^{j} y_{n-m_{j}}\right) \\
& \geq \limsup _{n \rightarrow \infty} y_{n}+\liminf _{n \longrightarrow \infty}\left(-\sum_{j=1}^{k} p_{n}^{j} y_{n-m_{j}}\right) \\
& \geq \limsup _{n \longrightarrow \infty} y_{n}-\limsup _{n \longrightarrow \infty}\left(\sum_{j=1}^{k} p_{n}^{j} y_{n-m_{j}}\right) \\
& \geq \limsup _{n \longrightarrow \infty} y_{n}-\sum_{j=1}^{k} \limsup _{n \longrightarrow \infty}\left(p_{n}^{j} y_{n-m_{j}}\right) \\
& \geq \limsup _{n \longrightarrow \infty} y_{n}-\sum_{j=1}^{k} \limsup _{n \longrightarrow \infty} p_{n}^{j} \limsup _{n \longrightarrow \infty} y_{n-m_{j}} \\
& \geq \limsup _{n \longrightarrow \infty} y_{n}\left(1-\sum_{j=1}^{k} \limsup _{n \longrightarrow \infty} p_{n}^{j}\right) \\
& \geq \limsup _{n \longrightarrow \infty} y_{n}(1-p) .
\end{aligned}
$$

Hence $\lim \sup _{n \rightarrow \infty} y_{n} \leq 0$, by (12), which implies the desired result $\lim _{n \rightarrow \infty} y_{n}=0$. If $y_{n}<0$ for $n>n_{1}$ then proceeding as above we can arrive at $\lim _{n \rightarrow \infty} y_{n}=0$. Thus the theorem is proved.

Theorem 2. Suppose that (H1)-(H4) hold. Assume that there exists a positive constant $p$ such that the sequences $\left\{p_{n}^{j}\right\}$ for $j=$ $1,2, \ldots, k$ satisfy the condition

$$
\begin{aligned}
& \qquad p_{n}^{j} \leq 0, \text { for every } j=1,2, \ldots, k \\
& \text { and } \sum_{j=1}^{k} \liminf _{n \longrightarrow \infty} p_{n}^{j}>-p>-1 .
\end{aligned}
$$

Then every solution of (1) oscillates or tends to zero as $n \longrightarrow \infty$.

Proof. Proceeding as in the proof of Theorem 1 and setting $z_{n}$, $w_{n}$ as in (13) and (14), respectively, we obtain (15) and further prove $y_{n}$ is bounded with $\lim \inf _{n \rightarrow \infty} y_{n}=0$. From (H3) and the fact that $w_{n}$ is monotonic it follows that $\lim _{n \rightarrow \infty} w_{n}=$ 
$\lim _{n \rightarrow \infty} z_{n}=\delta \in \mathbb{R}$. As $z_{n} \geq 0$, so $\delta \geq 0$. We claim $\delta=0$; if not then $\delta>0$, and this implies

$$
\begin{aligned}
\delta & =\liminf _{n \longrightarrow \infty} z_{n}=\liminf _{n \longrightarrow \infty}\left(y_{n}-\sum_{j=1}^{k} p_{n}^{j} y_{n-m_{j}}\right) \\
& \leq \liminf _{n \longrightarrow \infty} y_{n}+\limsup _{n \longrightarrow \infty}\left(-\sum_{j=1}^{k} p_{n}^{j} y_{n-m_{j}}\right) \\
& \leq \sum_{j=1}^{k} \limsup _{n \longrightarrow \infty}\left(-p_{n}^{j}\right) \limsup _{n \longrightarrow \infty}\left(y_{n-m_{j}}\right) \\
& =\sum_{j=1}^{k}-\liminf _{n \longrightarrow \infty}\left(p_{n}^{j}\right) \limsup _{n \longrightarrow \infty}\left(y_{n-m_{j}}\right) \\
& \leq p \limsup _{n \longrightarrow \infty}\left(y_{n}\right) \leq p \alpha .
\end{aligned}
$$

Hence we get

$$
\alpha \geq \frac{\delta}{p}>\delta
$$

Again

$$
\begin{aligned}
& \delta=\limsup _{n \rightarrow \infty} z_{n}=\limsup _{n \rightarrow \infty}\left(y_{n}-\sum_{j=1}^{k} p_{n}^{j} y_{n-m_{j}}\right) \\
& \geq \limsup _{n \rightarrow \infty} y_{n}+\liminf _{n \rightarrow \infty}\left(-\sum_{j=1}^{k} p_{n}^{j} y_{n-m_{j}}\right)
\end{aligned}
$$

$p_{n}^{j}<0$ for every $j=1,2, \ldots, k$ and there exists, $i \in\{1,2,3, \ldots, k\}$ such that $\limsup _{n \rightarrow \infty} p_{n}^{i}-\sum_{j \neq i} \liminf _{n \rightarrow \infty} p_{n}^{j}<-1$.

Then every solution of (1) oscillates or tends to zero as $n \longrightarrow \infty$.

Proof. Proceeding as in the proof of Theorem 1 and setting $z_{n}, w_{n}$ as in (13) and (14), respectively, we obtain (15) and further prove $y_{n}$ is bounded with $\liminf _{n \rightarrow \infty} y_{n}=0$. From (H3) and that $w_{n}$ is monotonic it follows that $\lim _{n \rightarrow \infty} w_{n}=$ $\lim _{n \rightarrow \infty} z_{n}=\delta \in \mathbb{R}$. As $z_{n} \geq 0$, so $\delta \geq 0$. We claim $\delta=0$. If not, then $\delta>0$, and this implies

$$
\begin{aligned}
\delta= & \liminf _{n \longrightarrow \infty} z_{n}=\liminf _{n \longrightarrow \infty}\left(y_{n}-\sum_{j=1}^{k} p_{n}^{j} y_{n-m_{j}}\right) \\
\leq & \limsup _{n \longrightarrow \infty}\left(y_{n}+\sum_{j \neq i}-p_{n}^{j} y_{n-m_{j}}\right) \\
& +\liminf _{n \longrightarrow \infty}\left(-p_{n}^{i} y_{n-m_{i}}\right) \\
\leq & \limsup _{n \longrightarrow \infty} y_{n}+\limsup _{n \longrightarrow \infty} \sum_{j \neq i}-p_{n}^{j} y_{n-m_{j}} \\
& +\limsup _{n \longrightarrow \infty}\left(-p_{n}^{j}\right) \liminf _{n \longrightarrow \infty}\left(y_{n-m_{j}}\right)
\end{aligned}
$$

$$
\begin{aligned}
& =\limsup _{n \longrightarrow \infty} y_{n}+\liminf _{n \rightarrow \infty}\left(\sum_{j=1}^{k}\left(-p_{n}^{j}\right) y_{n-m_{j}}\right) \\
& \geq \limsup _{n \longrightarrow \infty} y_{n}+\sum_{j=1}^{k} \liminf _{n \longrightarrow \infty}\left(\left(-p_{n}^{j}\right) y_{n-m_{j}}\right) \\
& \geq \limsup _{n \longrightarrow \infty} y_{n}+\sum_{j=1}^{k} \liminf _{n \longrightarrow \infty}\left(-p_{n}^{j}\right) \liminf _{n \longrightarrow \infty} y_{n-m_{j}} \\
& =\limsup _{n \rightarrow \infty} y_{n}=\alpha \text {, }
\end{aligned}
$$

a contradiction, due to inequality (24). Hence we conclude $\delta=0$ and from $z_{n}>y_{n}$, it follows that $\lim _{n \rightarrow \infty} y_{n} \leq 0$. Hence $\lim _{n \rightarrow \infty} y_{n}=0$.

The proof for the case $y_{n}<0$ for large $n$ is similar. Hence the theorem is proved.

Remark 3. Theorems 1 and 2 hold good for $k=0$ and $k=1$. Hence these results could be compared with results concerned with the difference equations (4), (5), (6), and (7).

Theorem 4. Suppose that (H1)-(H4) hold. Assume that there exists a positive constant $p$ such that the sequences $\left\{p_{n}^{j}\right\}$ for $j=$ $1,2, \ldots, k$ satisfy the condition

$$
\begin{aligned}
& \leq \underset{n \rightarrow \infty}{\limsup } y_{n}+\sum_{j \neq i 1} \limsup _{n \rightarrow \infty}\left(-p_{n}^{j}\right) \limsup _{n \rightarrow \infty}\left(y_{n-m_{j}}\right) \\
& \leq \limsup _{n \rightarrow \infty}\left(y_{n}\right)\left[1-\sum_{j \neq i} \liminf _{n \rightarrow \infty} p_{n}^{j}\right] .
\end{aligned}
$$

Again we have

$$
\begin{aligned}
& \delta= \limsup _{n \longrightarrow \infty} z_{n}=\limsup _{n \longrightarrow \infty}\left(y_{n}-\sum_{j=1}^{k} p_{n}^{j} y_{n-m_{j}}\right) \\
& \geq \liminf _{n \longrightarrow \infty} y_{n}+\limsup _{n \longrightarrow \infty}\left(-\sum_{j=1}^{k} p_{n}^{j} y_{n-m_{j}}\right) \\
&=\underset{n \longrightarrow \infty}{\limsup }\left(-p_{n}^{i} y_{n-m i}\right)+\liminf _{n \longrightarrow \infty} \sum_{j \neq i}\left(-p_{n}^{j} y_{n-m_{j}}\right) \\
& \geq \limsup _{n \longrightarrow \infty} y_{n-m_{j}} \liminf _{n \longrightarrow \infty}\left(-p_{n}^{i}\right) \\
& \quad+\sum_{j \neq i} \liminf _{n \longrightarrow \infty}\left(\left(-p_{n}^{j}\right) y_{n-m_{j}}\right)
\end{aligned}
$$




$$
\begin{aligned}
& \geq \limsup _{n \rightarrow \infty} y_{n}\left(-\limsup _{n \rightarrow \infty} p_{n}^{i}\right) \\
& +\sum_{j \neq i} \liminf _{n \longrightarrow \infty}\left(-p_{n}^{j}\right) \liminf _{n \longrightarrow \infty} y_{n-m_{j}} \\
& \geq \underset{n \rightarrow \infty}{\limsup } y_{n}\left(-\limsup _{n \rightarrow \infty} p_{n}^{i}\right) .
\end{aligned}
$$

From (27) and (28), it follows that

$$
\limsup _{n \rightarrow \infty} y_{n}\left(\left(\sum_{j \neq i} \liminf _{n \rightarrow \infty} p_{n}^{j}\right)-1-\limsup _{n \rightarrow \infty} p_{n}^{i}\right) \leq 0
$$

Using (26), we obtain $\lim _{n \rightarrow \infty} y_{n}=0$. Thus the theorem is proved.

Next, we intend to present a result where $p_{n}^{j}, j=$ $1,2,3, \ldots, k$, satisfy the following condition:

$$
p_{n}^{j}>0 \text { for every } j=1,2, \ldots, k \text { and there exists, } i \in\{1,2,3, \ldots, k\} \text { such that } \liminf _{n \longrightarrow \infty} p_{n}^{i}-\sum_{j \neq i} \limsup _{n \longrightarrow \infty} p_{n}^{j}>1 \text {. }
$$

For that purpose we give an example which would lead us to our next result.

Example 5. Consider the first-order neutral delay difference equation with several delays and variable coefficients

$$
\begin{gathered}
\Delta\left[y_{n}-\left(1+2^{-n}\right) y_{n-1}-\left(4+2^{-n}\right) y_{n-2}\right] \\
+2^{(2 n+1) / 3} y_{n-4}=0 .
\end{gathered}
$$

Note that $p_{n}^{j}$ satisfy (30) for the above neutral delay difference equation (31). This neutral delay difference equation has an unbounded solution $y_{n}=2^{n}$ tending to $\infty$ as $n \longrightarrow \infty$ unlike other results presented so far.

The above example is the motivating point to the statement of our next result. Since the proof is almost similar to that of Theorem 4 , it is omitted.

Theorem 6. Suppose that (H1)-(H4) hold. Assume that there exists a positive constant $p$ such that the sequences $\left\{p_{n}^{j}\right\}$ for $j=1,2, \ldots, k$ satisfy the condition (30). Then every bounded solution of (1) oscillates or tends to zero as $n \longrightarrow \infty$.

Remark 7. The above Theorems 4 and 6 hold for $k=1$ but not for $k=0$. Hence these results can be compared with results concerned with neutral delay difference equations (4) and (5).

Few examples are noted below to illustrate our results and establish its significance.

Example 8. Consider the first-order neutral delay difference equation

$$
\Delta\left(y_{n}-p_{n}^{1} y_{n-1}-p_{n}^{2} y_{n-4}\right)+\frac{97}{60 y_{n-2}^{3}}=0, \quad n \geq 5
$$

where

$$
p_{n}^{1}= \begin{cases}\frac{1}{4}, & \text { if } n \text { is odd } \\ \frac{1}{5}, & \text { if } n \text { is even, }\end{cases}
$$

and

$$
p_{n}^{2}= \begin{cases}\frac{1}{3}, & \text { if } n \text { is odd } \\ \frac{1}{2}, & \text { if } n \text { is even. }\end{cases}
$$

The neutral delay difference equation (32) satisfies all the conditions of Theorem 1. As such, it has an oscillatory solution $y_{n}=(-1)^{n}$.

Example 9. Consider the first-order inhomogeneous neutral delay difference equation

$$
\begin{gathered}
\Delta\left(y_{n}+p_{n}^{1} y_{n-4}+p_{n}^{2} y_{n-5}\right)+\frac{3}{4} 2^{n-4} y_{n-3}^{3} \\
=-\frac{3}{2^{n+1}}-\frac{3}{2^{2 n-2}}, \quad n \geq 5
\end{gathered}
$$

where $p_{n}^{1}=2^{-n}+1 / 16$ and $p_{n}^{2}=2^{-n}+1 / 32$. This neutral delay difference equation satisfies all the conditions of Theorem 2 . As such, it has a bounded positive solution $y_{n}=2^{-n}$ tending to zero as $n \longrightarrow \infty$. Note that, no result in the papers cited under reference can be applied to the neutral delay difference equations (32) and (35).

Remark 10. Results of $[3,9]$ cannot be applied to the delay difference equation (9), because the condition (10) is not satisfied. However, due to Remark 3, Theorem 1 can be applied to the delay equation (9) as all the conditions are satisfied and as such the delay equation has a positive bounded solution $e^{-n}$ tending to zero as $n \longrightarrow \infty$. Thus our work complements the work in $[3,9]$. Further, since we do not assume $G$ is nondecreasing, our Theorems 1, 2, 4, and 6 improve and generalize the results in [7].

\section{Necessary Conditions}

In this section we show that (H4) is necessary for every solution of (1) to be oscillatory or tending to zero as $n \longrightarrow \infty$. For this, we need the following lemma.

Lemma 11 (Krasnoselskii's fixed point theorem [13]). Let $X$ be a Banach space and $S$ be a bounded closed convex subset of 
$X$. Let $A, B$ be operators from $S$ to $X$ such that $A x+B y \in S$ for every pair of $x, y \in S$. If $A$ is a contraction and $B$ is completely continuous then the equation

$$
A x+B x=x
$$

has a solution in $S$.

Theorem 12. Assume that (H2) holds. Further, assume that one of the conditions of (12) and (22) hold. Then (H4) is a necessary condition for all solution of (1) to be oscillatory or tending to zero as $n \longrightarrow \infty$.

Proof. Suppose the condition (12) holds. The proof for the case when (22) holds would follow on similar lines. Assume for the sake of contradiction that $(\mathrm{H} 4)$ does not hold. Hence

$$
\sum_{n=n_{0}}^{\infty} q_{n}<\infty
$$

Thus, all we need to show is the existence of a bounded solution $y_{n}$ of (1) with $\liminf \operatorname{in}_{n \rightarrow \infty} y_{n}>0$. From (H2), we find a positive constant $c$ and a positive integer $n_{1}>n_{0}>0$ such that

$$
\left|F_{n}\right|<c \quad \text { for } n \geq n_{1} .
$$

Choose a positive constant $L$ such that $L \geq 5 c / 1-p$. Since $G \in C(\mathbb{R}, \mathbb{R})$, let

$$
\mu=\max \{|G(x)|: c \leq x \leq L\} .
$$

Let

$$
\eta=\max \left\{m_{1}, m_{2}, \ldots, m_{k}\right\} .
$$

Then using (37) one can fix $n_{2}>n_{1}$ such that for $n \geq n_{2}$ it follows that

$$
\mu \sum_{i=n}^{\infty} q_{i}<c
$$

Choose $N_{1}>n_{2}$ such that

$$
N_{0}=\min \left\{\sigma\left(N_{1}\right), N_{1}-\eta\right\} .
$$

Let $X=\ell_{\infty}^{N_{0}}$, Banach space of real bounded sequences $x=$ $\left\{x_{n}\right\}$ with $x_{1}=x_{2}=\cdots=x_{N_{0}}$ and supremum norm

$$
\|x\|=\sup \left(\left|x_{n}\right|: n \geq N_{0}\right) \text {. }
$$

Define

$$
S=\left\{y \in X: c \leq y_{n} \leq L, n \geq N_{0}\right\} .
$$

Clearly $\mathrm{S}$ is a bounded closed and convex subset of $\mathrm{X}$. Now we define two operators $A$ and $B: S \longrightarrow X$ as follows. For $y \in S$, define

$$
\begin{aligned}
& (A y)_{n}= \begin{cases}(A y)_{N_{1}}, & N_{0} \leq n \leq N_{1} \\
\sum_{j=1}^{k} p_{n}^{j} y_{n-m_{j}}+F_{n}+3 c, & n \geq N_{1} .\end{cases} \\
& (B y)_{n}= \begin{cases}(B y)_{N_{1}}, & N_{0} \leq n \leq N_{1} \\
\sum_{i=n}^{\infty} q_{i} G\left(y_{\sigma(i)}\right), & n \geq N_{1} .\end{cases}
\end{aligned}
$$

First we show that if $x, y \in S$ then $A x+B y \in S$. Hence, for $x=\left\{x_{n}\right\}$ and $y=\left\{y_{n}\right\} \in S$ and for $n \geq N_{1}$ we obtain

$$
\begin{aligned}
(A x)_{n}+(B y)_{n} \leq & \sum_{j=1}^{k} p_{n}^{j} x_{n-m_{j}}+3 c+\sum_{i=n}^{\infty} q_{i}\left|G\left(y_{\sigma(i)}\right)\right| \\
& +\left|F_{n}\right| \leq p L+5 c \leq L .
\end{aligned}
$$

On the other hand

$$
\begin{aligned}
(A x)_{n}+(B y)_{n} & \geq 3 c-\sum_{i=n}^{\infty} q_{i}\left|G\left(y_{\sigma(i)}\right)\right|-\left|F_{n}\right| \\
& \geq 3 c-c-c \geq c .
\end{aligned}
$$

Hence

$$
c \leq(A x)_{n}+(B y)_{n} \leq L \text { for } n \geq N_{1} .
$$

Thus, we proved that $A x+B y \in S$ for any $x, y \in S$. Next we show that $A$ is a contraction on $S$. In fact for $x, y \in S$ and $n \geq N_{1}$ we have

$$
\begin{aligned}
\left\|(A x)_{n}-(A y)_{n}\right\| & \leq \sum_{j=1}^{k}\left|p_{n}^{j}\right|\left|x_{n-m_{j}}-y_{n-m_{j}}\right| \\
& \leq p\|x-y\|
\end{aligned}
$$

This implies $A$ is a contraction because $0<p<1$. Next we show that $B$ is completely continuous. For this as a first step we show that $B$ is continuous. Suppose the sequence $x^{l} \equiv\left\{x_{n}^{l}\right\} \longrightarrow x \equiv\left\{x_{n}\right\}$ in $S$ as $l \longrightarrow \infty$ (with $l$ taken from the index set). Since $S$ is closed then $x \in S$. For $n \geq N_{1}$ we have

$$
\left|\left(B x^{l}\right)_{n}-(B x)_{n}\right| \leq \sum_{i=n}^{\infty} q_{i}\left|G\left(x_{\sigma(i)}^{l}\right)-G\left(x_{\sigma(i)}\right)\right|
$$

Since $G$ is continuous, therefore $\left|G\left(x_{\sigma(i)}^{l}\right)-G\left(x_{\sigma(i)}\right)\right| \longrightarrow 0$ as $l \longrightarrow \infty$. Hence $B$ is continuous. Next what remained to show is $B S$ is relatively compact. Using the result [14, Theorem 3.3], we need only show that $B S$ is uniformly cauchy. Let $x \equiv\left\{x_{n}\right\}$ be a sequence in S. From (H2) and (37), it follows that, for $\epsilon>0$, there exists $N^{*} \geq N_{1}$ such that, for $n \geq N^{*}$,

$$
\sum_{i=n}^{\infty} q_{i}\left|G\left(x_{\sigma(i)}\right)\right|<\frac{\epsilon}{2} \text {. }
$$

Then for $n_{3}>n_{4} \geq N^{*}$ we have

$$
\left|(B x)_{n_{3}}-(B x)_{n_{4}}\right|<\epsilon .
$$

Thus BS is uniformly cauchy. Hence it is relatively compact. Then by Lemma 11, we can find $x^{0}$ in $S$ such that $A x^{0}+B x^{0}=$ $x^{0}$. Clearly, $\left(x^{0}\right)_{n}$ is a bounded, positive solution of (1) with limit infimum greater than or equal to $c>0$. Thus the theorem is proved.

Theorem 13. Assume that (H2) holds. Further assume that one of the conditions of (26) and (30) holds. Then (H4) is a necessary condition for all solution of (1) to be oscillatory or tending to zero as $n \longrightarrow \infty$. 
Proof. Suppose that $p_{n}$ satisfies (30). The proof for the case when (26) holds is similar. Assume for the sake of contradiction that (H4) does not hold. Hence (37) holds. Thus, all we need to show is the existence of a bounded solution $y_{n}$ of (1) with $\liminf \operatorname{in}_{n \rightarrow \infty} y_{n}>0$. From (H2), we find a positive constant $L$ and a positive integer $n_{1}>n_{0}>0$ such that

$$
\left|F_{n}\right|<L \quad \text { for } n \geq n_{1} \text {. }
$$

By (30), we can find a small positive real $\epsilon$, a lower bound $c$ for $p_{n}^{i}$, and upper bounds $d_{j}$ for $p_{n}^{j}(j \neq i$ and $1 \leq j \leq k)$ such that $c-\sum d_{j}-1=\epsilon$. Let $\sum d_{j}=d$. Hence $c=d+1+\epsilon$. Next choose an upper bound $b$ for $p_{n}^{i}$ such that $b<\left(c^{2}-c\right) / d$. The nonexistence of such an upper bound for $p_{n}^{i}$ would lead to the fact that, for all $\delta>0, b=c+\delta$ and $b \geq\left(c^{2}-c\right) / d$. Taking $\delta=\epsilon$, we have $\epsilon^{2}+\epsilon \leq 0$, a contradiction. Choose a real $\lambda$ as follows:

$$
0<\lambda=\frac{(L+\epsilon) d+(c-1)(c+L+\epsilon)}{c^{2}-(b d+c)} .
$$

Let

$$
H=\frac{b \lambda+L+\epsilon}{c-1}
$$

From (54) and (55) it follows that

$$
\lambda-\frac{H d+L+\epsilon}{c}=1 .
$$

Since $G \in C(\mathbb{R}, \mathbb{R})$, let

$$
\mu=\max \{|G(x)|: 1 \leq x \leq H\} .
$$

Let $\eta=\max \left\{m_{1}, m_{2}, \ldots, m_{k}\right\}$. Then using (37), one can fix $n_{2}>n_{1}$ such that for $n \geq n_{2}$ it follows that

$$
\mu \sum_{i=n}^{\infty} q_{i}<\epsilon .
$$

Choose $N_{1}>n_{2}$ such that

$$
N_{0}=\min \left\{\sigma\left(N_{1}\right), N_{1}-\eta\right\} .
$$

Let $X=\ell_{\infty}^{N_{0}}$, Banach space of real bounded sequences $x=$ $\left\{x_{n}\right\}$ with $x_{1}=x_{2}=\cdots=x_{N_{0}}$ and supremum norm

$$
\|x\|=\sup \left(\left|x_{n}\right|: n \geq N_{0}\right) \text {. }
$$

Define

$$
S=\left\{y \in X: 1 \leq y_{n} \leq H, n \geq N_{0}\right\} .
$$

Clearly $\mathrm{S}$ is a bounded closed and convex subset of $\mathrm{X}$. Now we define two operators $A$ and $B: S \longrightarrow X$ as follows. For $y \in S$, define

$$
\begin{aligned}
& (A y)_{n}= \begin{cases}(A y)_{N_{1}}, & N_{0} \leq n \leq N_{1} \\
\frac{y_{n+m_{i}}}{p_{n+m_{i}}^{i}}-\frac{\sum_{j \neq i} p_{n+m_{i}}^{j} y_{n-m_{j}+m_{i}}}{p_{n+m_{i}}^{i}}+\frac{b \lambda}{p_{n+m_{i}}^{i}}-\frac{F_{n+m_{i}}}{p_{n+m_{i}}^{i}}, & n \geq N_{1} .\end{cases} \\
& (B y)_{n}= \begin{cases}(B y)_{N_{1}}, & N_{0} \leq n \leq N_{1} \\
\frac{-\sum_{j=n+m_{i}}^{\infty} q_{j} G\left(y_{\sigma(j)}\right)}{p_{n+m_{i}}^{i}}, & n \geq N_{1} .\end{cases}
\end{aligned}
$$

Proceeding as in the proof of above theorem we show that (i) if $x, y \in S$ then $A x+B y>1$ by (56) and $A x+B y<H$ by (55), so that $A x+B y \in S$, (ii) $\left\|A x_{n}-A y_{n}\right\|<[(d+1) / c] \| x_{n}-$ $y_{n} \|$, hence $A$ is a contraction on $S$, and (iii) $B$ is completely continuous. This completes the proof of the theorem.

Remark 14. For the results in this section, we assume none of conditions (H3), $G$ is nondecreasing, and $x G(x)>0$, whereas the authors $[7,8]$ assumed these three conditions in their corresponding results. Hence the results of this article generalize and improve the corresponding results of these papers.

Combining all the above results, i.e., Theorems 1, 2, 4, 6, 12 , and 13 , we obtain the following theorem.
Theorem 15. Suppose that (H1)-(H3) hold. Assume $p_{n}^{j}$ in (1) to satisfy one of the four conditions (12), (22), (26), and (30). Then (H4) is both necessary and sufficient condition for every solution of (1) to be oscillatory or tending to zero as $n \longrightarrow \infty$.

Remark 16. The results of this work hold for $G(x)=x$ and $f(x)=0$, i.e., for the linear homogeneous equation associated with (1).

\section{Data Availability}

Previously reported data were used to support this study and are available at [DOI or OTHER PERSISTENT IDENTIFIER]. 
These prior studies (and datasets) are cited at relevant places within the text as references [\#-\#].

\section{Disclosure}

This work is done for the Ph.D. thesis work of the second author.

\section{Conflicts of Interest}

The authors declare that they have no conflicts of interest.

\section{Acknowledgments}

The authors are thankful to Professor Prayag Prasad Mishra for his valuable guidance during the completion of this paper.

\section{References}

[1] I. Gyori and G. Ladas, Oscillation Theory of Delay-Differential Equations with Applications, Clarendon Press, Oxford, UK, 1991, https:/global.oup.com/academic/product/oscillation-theory-ofdelay-differential-equations-9780198535829?lang=en\&cc=es.

[2] G. E. Chatzarakis and G. N. Miliaras, "Asymptotic behavior in neutral difference equations with variable coefficients and more than one delay arguments," Journal of Mathematical and Computational Science, vol. 1, no. 1, pp. 32-52, 2011, http://scik.org/ index.php/jmcs/article/view/16.

[3] R. Koplatadze and S. Pinelas, "Oscillation Criteria for the First-Order Linear Difference Equations with Several Delay Arguments," Journal of Mathematical Sciences, vol. 208, no. 5, pp. 571-592, 2015.

[4] R. Koplatadze, "On asymptotic behavior of solutions of first order difference equations with several delay," Bulletin. Tbilisi International Centre of Mathematics and Informatics, vol. 21, no. 2, pp. 117-123, 2017, https://www.emis.de/journals/TICMI/ vol21_2/p117-121.pdf.

[5] R. P. Agarwal, Difference Equations and Inequalities, Marcel Dekker, New York, NY. USA, 2nd edition, 2000, https://doc .lagout.org/science/0_Computer\%20Science/3_Theory/Mathematics/Difference\%20Equations\%20and\%20Inequalities\%20\%20Theory.pdf.

[6] R. N. Rath, N. Misra, and S. K. Rath, "Sufficient conditions for oscillatory behaviour of a first order neutral difference equation with oscillating coefficients," Acta Mathematica Academiae Paedagogicae Nyiregyhaziensis, vol. 25, no. 1, pp. 55-63, 2009, https://www.emis.de/journals/AMAPN/vol25_1/7.html.

[7] N. Parhi and A. K. Tripathy, "Oscillation of forced nonlinear neutral delay difference equations of first order," Czechoslovak Mathematical Journal, vol. 53, no. 1, pp. 83-101, 2003.

[8] N. Parhi and R. N. Rath, "Oscillation criteria for forced first order neutral differential equations with variable coefficients," Journal of Mathematical Analysis and Applications, vol. 256, no. 2, pp. 525-541, 2001.

[9] G. E. Chatzarakis, R. Koplatadze, and I. P. Stavroulakis, "Optimal oscillation criteria for first order difference equations with delay argument," Pacific Journal of Mathematics, vol. 235, no. 1, pp. 15-33, 2008, https://msp.org/pjm/2008/235-1/pjm-v235-n1-p02-p.pdf.

[10] R. N. Rath, S. Padhi, and B. L. Barik, "Oscillatory and asymptotic behaviour of a homogeneous neutral delay difference equation of second order," Bulletin of the Institute of Mathematics, Academia Sinica, vol. 3, no. 3, pp. 453-467, 2008, https://web.math.sinica.edu.tw/bulletin_ns/20083/2008310.pdf.

[11] E. Thandapani, R. Arul, and P. S. Raja, "Oscillatory and asymptotic behavior of solutions of non-homogeneous neutral difference equations," Studies of the University of Zilina Mathematical Series, vol. 15, no. 1, pp. 67-82, 2002, https://frcatel.fri.uniza.sk/ studies/.

[12] E. Thandapani, R. Arul, and P. . Raja, "Oscillation of first order neutral delay difference equations," Applied Mathematics ENotes, vol. 3, pp. 88-94, 2003, https://www.emis.de/journals/ AMEN/.

[13] L. H. Erbe, Q. K. Kong, and B. G. Zhang, Oscillation Theory for Functional Differential Equations, Marcel Dekker, New York, NY, USA, 1995, https://www.hindawi.com/journals/aaa/2011/ 591254/ref/.

[14] S. S. Cheng and W. T. Patula, "An existence theorem for a nonlinear difference equation," Nonlinear Analysis. Theory, Methods \& Applications, vol. 20, no. 3, pp. 193-203, 1993. 


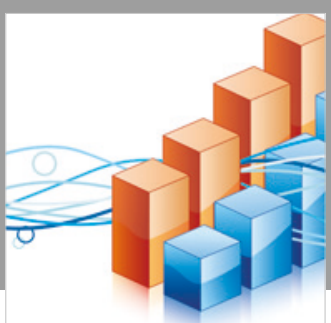

Advances in

Operations Research

\section{-n-m}
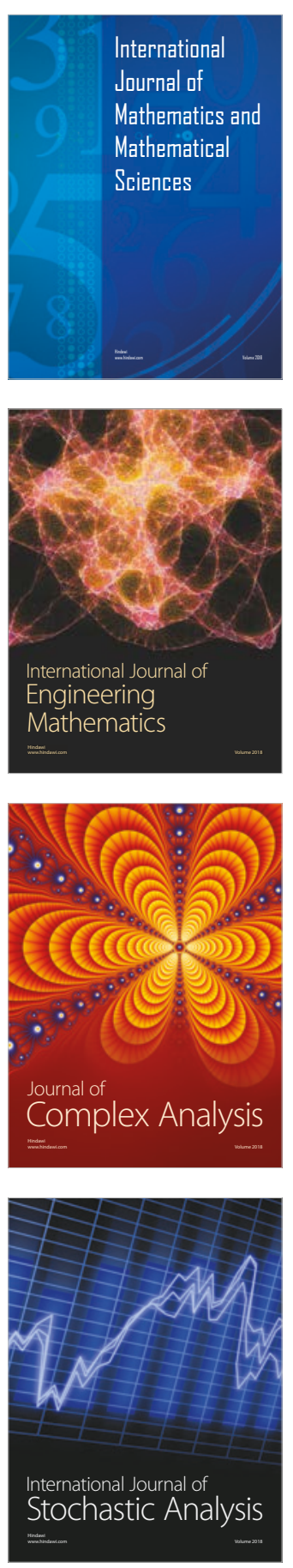
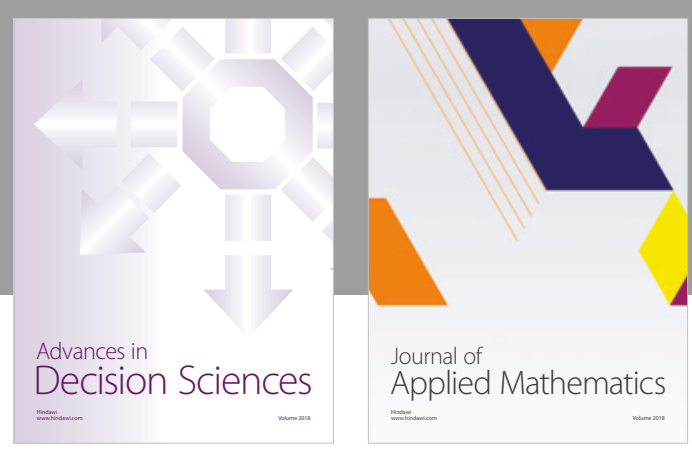

Journal of

Applied Mathematics
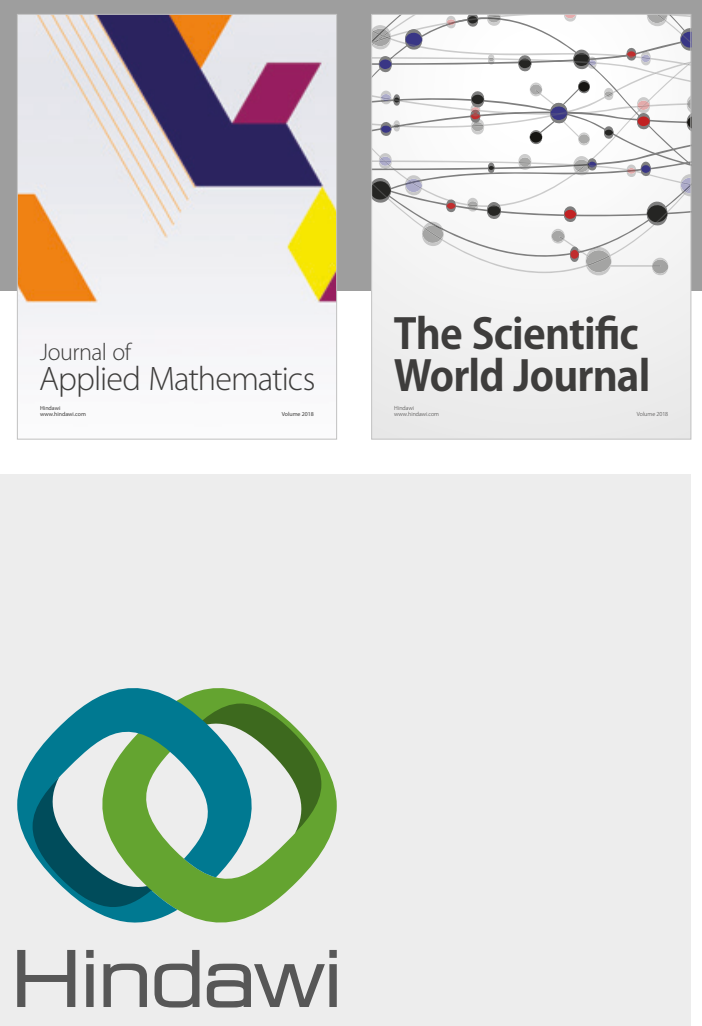

Submit your manuscripts at

www.hindawi.com

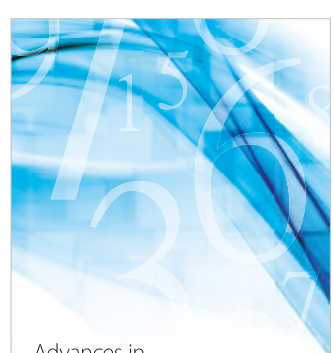

Advances in
Numerical Analysis
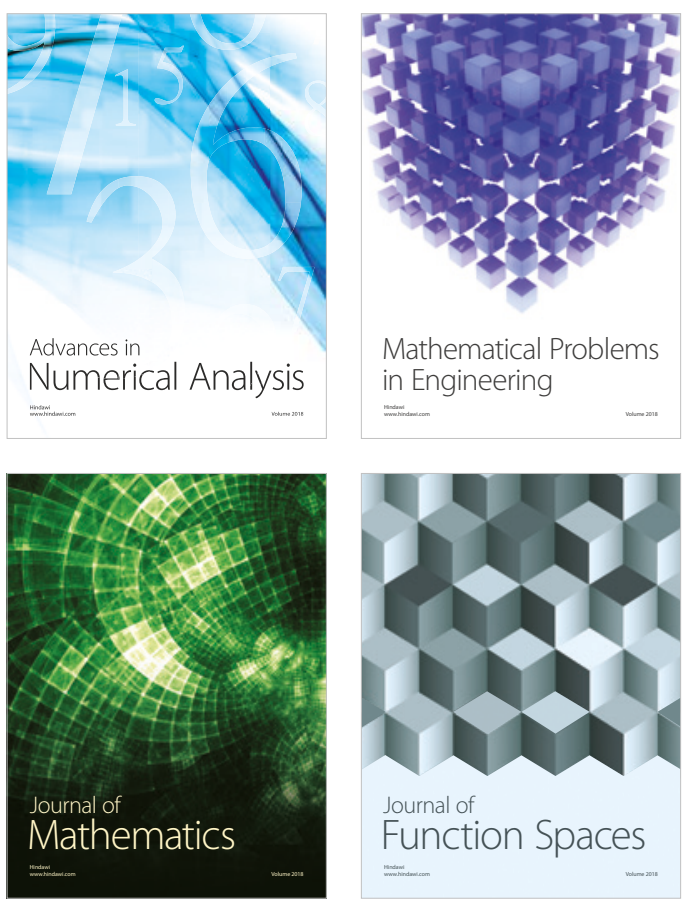

Mathematical Problems in Engineering

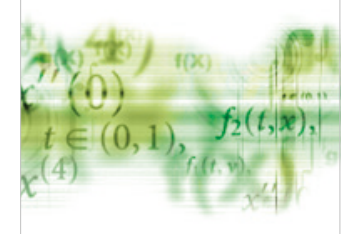

International Journal of

Differential Equations

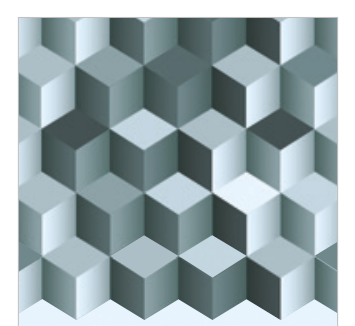

Journal of

Function Spaces

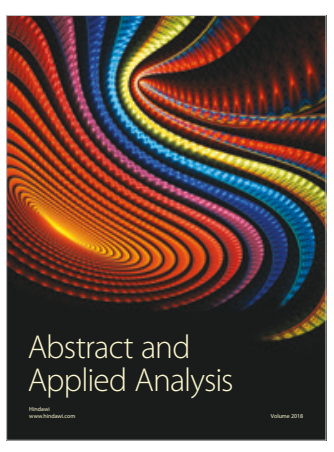

The Scientific

World Journal

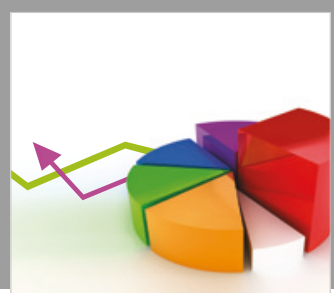

Journal of

Probability and Statistics
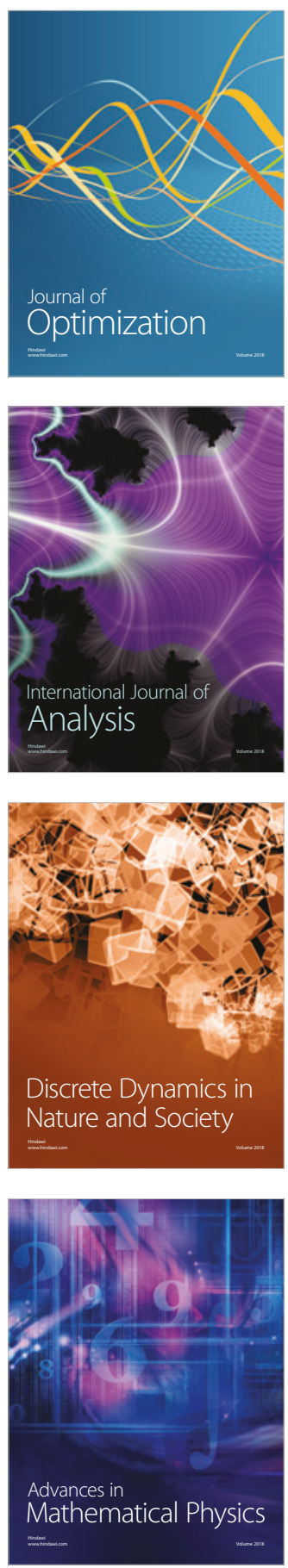